\title{
ANALYTICAL REPRESENTATION OF THE LANG-KOHN DENSITY PROFILES BY THE NUMERICAL FITTING*
}

\author{
J.M. RogowSKA, K.F. WoJCIECHOWSKI \\ Institute of Experimental Physics, University of Wroclaw \\ Pl. Maxa Borna 9, 50-204 Wrocław, Poland \\ AND M. MACIEJEWSKI \\ Institute of Informatics, University of Wrocław \\ Przesmyckiego 20, 50-204 Wrocław, Poland \\ (Received June 18, 1993; in final form October 26, 1993)

\begin{abstract}
The numerical fitting of an analytical function representing electron density profile at a jellium surface to the one tabulated by Lang and Kohn is presented. The two sets of parameters entering the electron density profile is proposed. The first one is obtained by purely numerical fitting, and the second one is calculated under condition that electron density profile must satisfy the Budd-Vannimenus theorem. The obtained parameters are given as analytical functions of the Wigner-Seitz radius $r_{\mathrm{s}}$ describing mean electron density $\bar{n}$ in a metal $\left(\bar{n}^{-1}=4 / 3 \pi r_{s}^{3}\right)$. The comparison of presented electron density profile with variational trial function given by Perdew is also discussed.
\end{abstract}

PACS numbers: $73.30 .+y, 73.20 .-r$

\section{Introduction}

The surface properties of the simple metals are mainly determined by the electron density profile (EDP) $n(x)$ and the potential barrier $V(x)$ at the surface.

The simple metals are the ones which appear in group IA, IIA, IIIB and IVB and are bounded by $s$ and $p$ valence electrons. The $s$ and $p$ orbitals are very spread out and interact in the metal to give wide overlapping bands which are a recognizable small distribution of free-electron bands [1].

Lang and Kohn [2] were the first who, using the local density approximation for exchange and correlation, determined self-consistently $n(x)$ and $V(x)$, giving tabulated values of these functions calculated numerically for the Wigner-Seitz

*This work was supported by the Wrocław University contract 1010/S/IFD/92. 
parameter $r_{\mathrm{s}}=(9 \pi / 4)^{1 / 3} k_{\mathrm{F}}^{-1}$ values $2,3,4,5$ and 6 bohrs $^{\dagger}$, in the interval $(-1,1)$ of the distance $x$ (in $2 \pi / k_{\mathrm{F}}$ units where $k_{\mathrm{F}}$ is the Fermi wave number) from the surface of the positive background. The numerical results of Lang and Kohn (LK) were widely explored as a reference point for other calculations, mainly done non-self-consistently $[3-5]^{\ddagger}$ In many cases, however, the analytical forms of $n(x)$ and $V(x)$ are needed, especially for practical purposes.

The effective potential $V(x)$ of Lang and Kohn was fitted by saturated image barrier [6] and more recently by the analytical potentials given by Mola et al. [7] which reproduce the first peak in the potential of LK (which is a Friedel oscillation) and approach the classical image potential for large separation from the surface as well.

The numerous attempts of approximation of the LK numerical values of EDP by simple one or two parameters trial functions [8] was also made. The parameters entering the trial EDP functions were usually determined by the charge neutrality conditions, minimum of the surface energy or the Budd-Vannimenus theorem (BVT) [9], or both these conditions. The analytical formula for $n(x)$ approximating properly charge densities tabulated by LK may enable to perform the calculations of a surface properties of metals. For instance, the important problem of the gradient corrections to the exchange-correlation energy of electron at metal surfaces needs such a formula [10]. In this context we give in the present paper the analytic expression for $n(x)$, which is numerically fitted function to the results of LK.

\section{The model electron density profile}

As it is well known, the semi-infinite metal $(0<x)$ may be simulated by the electrons in the finite potential barrier at $x=0$ with sine-wave form of the wave functions $\psi_{k}(x)=\sin [k x-\gamma(k)], \gamma$ being the phase shift, thus the electron density

$$
n(x)=\frac{1}{\pi^{2}} \int_{0}^{k_{\mathrm{F}}}\left(k_{\mathrm{F}}^{2}-k^{2}\right) \psi_{k}^{2}(x) \mathrm{d} k
$$

for $x \rightarrow-\infty$, has the form [2]:

$$
n(x)_{x \rightarrow-\infty} \approx \bar{n}\left\{1+\frac{3 \xi \cos 2\left[\left(k_{\mathrm{F}} x-\gamma\left(k_{\mathrm{F}}\right)\right]\right.}{\left(2 k_{\mathrm{F}} x\right)^{2}}\right\}+\mathrm{O}\left(\frac{1}{x^{3}}\right)
$$

where $\xi \approx 1$ and $\bar{n}$ is the electron density of a bulk metal. The above result can be obtained by inserting the sine form of the wave function into Eq. (1).

In order to approximate the charge densities tabulated by LK by an analytic function $n(x)$ we propose this function in the form

$$
\nu(x) \equiv \frac{n(x)}{\vec{n}}= \begin{cases}1+\frac{3 \xi \cos 2\left[\left(k_{\mathrm{F}} x-\gamma\left(k_{\mathrm{F}}\right)\right]\right.}{\left(2 k_{\mathrm{F}} x\right)^{2}}, & x \leq x_{1}<0 \\ \sum_{i=0}^{3} a_{i}\left(x-x_{1}\right)^{i}, & x_{1}<x<x_{2} \\ \lambda \mathrm{e}^{-\beta x}, & x \geq x_{2}>0\end{cases}
$$

t The atomic units are used unless otherwise stated. In this system the unit of energy is 1 hartree $=27.21 \mathrm{eV}$.

$\ddagger$ The expressions (9) and (11) given in Ref. [4] are incorrect, Eq. (9) should be $\xi\left(r_{s}\right)=0.50306-0.026357 r_{s}+0.0005357143 r_{s}^{2}$ and Eq. (11) has the form $\sigma(\gamma)=$ $\frac{k_{\mathrm{F}}^{9 / 2}}{27 \pi^{2}}\left[\frac{a_{e s}}{3 \gamma^{3}}+\frac{1}{\gamma}\left(-a_{0}+\frac{a_{x}}{k_{\mathrm{F}}}+\frac{a_{c}(\bar{n})}{k_{\mathrm{F}}}\right)+\frac{a_{2 \gamma}}{k_{\mathrm{F}}}+\frac{a_{4} \gamma^{3}}{3 k_{\mathrm{F}}^{2}}\right]$. 
where $a_{i}$ with $i=0,1,2,3, \gamma$ and $\beta$ are parameters to determine.

In order to warrant that $\nu(x)$ will be continuous with its first derivative, we put

$$
\begin{aligned}
& a_{0}=1+\frac{3 \xi \cos \left(x_{1}^{\prime}-2 \gamma\right)}{x_{1}^{\prime 2}}, \\
& a_{1}=\frac{-2 k_{F}}{x_{1}^{\prime}}\left[2\left(a_{0}-1\right)+\frac{3 \xi \sin \left(x_{1}^{\prime}-2 \gamma\right)}{x_{1}^{\prime}}\right], \\
& a_{2}=-\frac{1}{h^{2}}\left[3 a_{0}+2 h a_{1}-\lambda(3+\beta h) \mathrm{e}^{-\beta x_{2}}\right], \\
& a_{3}=\frac{1}{h^{3}}\left[2 a_{0}+h a_{1}-\lambda(2+\beta h) \mathrm{e}^{-\beta x_{2}}\right],
\end{aligned}
$$

where

$$
x_{1}^{\prime}=2 k_{\mathrm{F}} x_{1}, \quad h=x_{2}-x_{1} .
$$

\section{Numerical approximations}

\subsection{Approximation 1 (purely numerical)}

The parameters $\xi, \gamma, x_{1}, x_{2}, \lambda$ and $\beta$ are fitted by the least squares method for every point given by LK (Table I, in [2]) for $r_{\mathrm{s}}=2,3,4,5$ and 6 . Table I contains these parameters, together with the values of the standard deviations $\sigma$.

In practice, the simple metals are described by the values of $r_{\mathrm{s}}$ which are real numbers, not the integer ones like in LK tables. Therefore, we approximate these parameters by the linear or quadratic function of $r_{\mathrm{s}}$ :

$$
\begin{aligned}
& \xi\left(r_{\mathrm{s}}\right)=-0.019 r_{\mathrm{s}}^{2}+0.3098 r_{\mathrm{s}}-0.5281, \\
& \gamma\left(r_{\mathrm{s}}\right)=0.0615 r_{\mathrm{s}}+0.897, \\
& x_{1}\left(r_{\mathrm{s}}\right)=-0.6441 r_{\mathrm{s}}-0.6892, \\
& x_{2}\left(r_{\mathrm{s}}\right)=0.1261 r_{\mathrm{s}}+0.508, \\
& \lambda\left(r_{\mathrm{s}}\right)=0.002643 r_{\mathrm{s}}^{2}-0.0456 r_{\mathrm{s}}+0.5836, \\
& \beta\left(r_{\mathrm{s}}\right)=0.02714 r_{\mathrm{s}}^{2}-0.3229 r_{\mathrm{s}}+1.6474 .
\end{aligned}
$$

The above approximation, denoted further as 1 , is purely numerical one without any additional conditions imposed on the function (3). It does not satisfy the charge neutrality condition (ChNC) (see Eq. (15)). The integral $I=10^{-2}$ for $4.5 \geq r_{\mathrm{s}}>3$, and for the remained values of $r_{\mathrm{s}}$ we have $I=10^{-1}$. 


\subsection{Approximation 2 (charge neutrality condition, the Budd-Vannimenus theorem)}

The ChNC for jellium model

$$
I \equiv \int_{-\infty}^{\infty}[\nu(x)-\theta(-x)] \mathrm{d} x=0,
$$

where $\theta(-x)$ is the Heaviside step function, is of course satisfied in the LK self-consistent procedure, therefore we decided to perform the approximation 2, which assumes equality (15).

In this approximation, we decided to introduce the BVT [9]:

$$
\phi(0)-\phi(-\infty)=\bar{n} \frac{\mathrm{d} \varepsilon_{\mathrm{T}}(\bar{n})}{\mathrm{d} \bar{n}},
$$

as the additional condition. The $\varepsilon_{\mathrm{T}}(\bar{n})$ is the sum of the kinetic, exchange, and correlation energies per particle for the uniform electron gas of density $\bar{n}$, and the electrostatic potential $\phi(x)$ is connected with $\nu(x)$ by the Poisson equation

$$
\phi(x)=\phi(-\infty)+4 \pi \bar{n} \int_{-\infty}^{x}\left(x^{\prime}-x\right)\left[\nu\left(x^{\prime}\right)-\theta\left(-x^{\prime}\right)\right] \mathrm{d} x^{\prime} .
$$

The LK results do not satisfy the BVT, in particular for low metallic densities $[9,11]$, therefore it is not surprising that the approximation 2 does not reproduce the LK results as exactly as the approximation 1 (compare Tabs. I and II).

\section{TABLE I}

The values of the parameters of the function $\nu(x)$ (Eq. (3)) fitted to the LK values of $\nu(x)$ within approximation 1 by the least squares method together with the values of the standard deviation $\sigma$ (in $\bar{n}$ units).

\begin{tabular}{c|c|c|c|c|c|c|c}
\hline \hline$r_{\mathrm{s}}$ & $\xi$ & $\gamma$ & $x_{1}$ & $x_{2}$ & $\lambda$ & $\beta$ & $\sigma \times 10^{3}$ \\
\hline 2 & 0.10414 & 1.87862 & -2.13917 & 0.33884 & 0.47926 & 1.06807 & 1.15 \\
3 & 0.23038 & 1.07406 & -2.62534 & 0.87228 & 0.46897 & 0.90810 & 0.79 \\
4 & 0.40869 & 1.15320 & -3.07917 & 1.00638 & 0.44383 & 0.79373 & 0.62 \\
5 & 0.54525 & 1.21317 & -3.90806 & 1.14940 & 0.42284 & 0.72218 & 0.92 \\
6 & 0.64756 & 1.25737 & -4.65080 & 1.27936 & 0.403957 & 0.68092 & 1.43
\end{tabular}
form:

The ChNC (Eq. (15)) is satisfied exactly, if the parameter $\lambda$ has the following

$$
\lambda\left(\xi, \gamma, x_{1}, x_{2}, \beta\right)=-\frac{s+x_{1}+h\left(\frac{1}{2} a_{0}+\frac{1}{12} h a_{1}\right)}{\mathrm{e}^{-\beta x_{2}}\left[h\left(\frac{1}{2}+\frac{1}{12} \beta h\right)+\frac{1}{\beta}\right]},
$$

where

$$
s=\frac{3 \xi}{2 k_{\mathrm{F}}}\left[-\frac{\cos \left(x_{1}^{\prime}-2 \gamma\right)}{x_{1}^{\prime}}+\cos (2 \gamma) \operatorname{Si}\left(-x_{1}^{\prime}\right)+\sin (2 \gamma) \operatorname{Ci}\left(-x_{1}^{\prime}\right)\right],
$$


TABLE II

The values of the parameters of the function $\nu(x)$ (Eq. (3)) fitted to the LK values of $\nu(x)$ within approximation 2 by the least squares method together with the values of the standard deviation $\sigma$ (in $\bar{n}$ units).

\begin{tabular}{c|c|l|c|c|c|c|c}
\hline \hline$r_{\mathrm{s}}$ & $\xi$ & \multicolumn{1}{|c|}{$\gamma$} & $x_{1}$ & $x_{2}$ & $\lambda$ & $\beta$ & $\sigma \times 10^{3}$ \\
\hline 2 & 0.13617 & 2.14783 & -2.12447 & 0.83272 & 0.53300 & 1.14708 & 3.18 \\
3 & 0.21178 & 1.097523 & -2.70340 & 0.96173 & 0.48498 & 0.94596 & 0.98 \\
4 & 0.40383 & 1.14011 & -3.07833 & 1.05737 & 0.45719 & 0.82430 & 0.77 \\
5 & 0.52710 & 1.20460 & -3.76909 & 1.22829 & 0.43830 & 0.74412 & 1.29 \\
6 & 0.62162 & 1.235112 & -4.46780 & 1.36883 & 0.41994 & 0.67399 & 3.41
\end{tabular}

$$
\operatorname{Si}(z)=-\int_{z}^{\infty} \frac{\sin x}{x} \mathrm{~d} x, \quad \operatorname{Ci}(z)=-\int_{z}^{\infty} \frac{\cos x}{x} \mathrm{~d} x
$$

and the parameters $x_{1}^{\prime}, h, a_{0}, \ldots, a_{3}$ are defined by Eqs. (4) $\div(8)$.

The condition (16) is fulfilled with high accuracy through choosing the remainder parameters in such a way to minimize the function

$$
F\left(\xi, \gamma, x_{1}, x_{2}, \beta\right)=\sum_{i}\left[\nu_{i}-\nu\left(x_{i}\right)\right]^{2}+w \delta^{2},
$$

where

$$
\delta=\phi(0)-\phi(-\infty)-\bar{n} \frac{\mathrm{d} \varepsilon_{\mathrm{T}}(\bar{n})}{\mathrm{d} \bar{n}} .
$$

The weight $w$ was chosen to warrant the accuracy of fulfilling BVT to $\frac{\delta}{\bar{n}} \frac{\mathrm{d} \varepsilon_{\mathrm{T}}(\bar{n})}{\mathrm{d} \bar{n}} \leq 10^{-6}$.

The obtained in the framework of approximation 2 parameters are collected in Table II. Additionally, we approximate these parameters by the linear or quadratic function of $r_{\mathrm{s}}$ in the following way:

$$
\begin{aligned}
& \xi\left(r_{\mathrm{s}}\right)=-0.02325 r_{\mathrm{s}}^{2}+0.34275 r_{\mathrm{s}}-0.60075 \\
& \gamma\left(r_{\mathrm{s}}\right)=0.0469 r_{\mathrm{s}}+0.9602, \\
& x_{1}\left(r_{\mathrm{s}}\right)=-0.5938 r_{\mathrm{s}}-0.8964 \\
& x_{2}\left(r_{\mathrm{s}}\right)=0.1341 r_{\mathrm{s}}+0.547 \\
& \beta\left(r_{\mathrm{s}}\right)=0.0229286 r_{\mathrm{s}}^{2}-0.3015286 r_{\mathrm{s}}+1.658
\end{aligned}
$$

\section{Discussion and conclusions}

The comparison of computed electron density profiles with the LK results may be done by discussing the values of standard deviations $\sigma$ given in Tabs. I and II. Generally, standard deviations of approximation 1 are smaller than of 


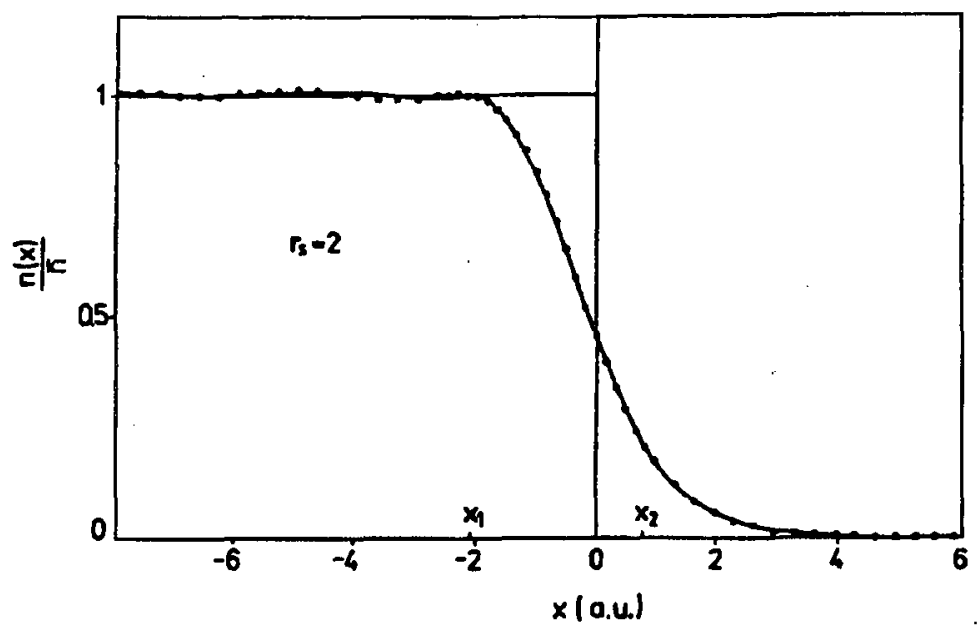

Fig. 1. The comparison of EDP obtained from Lang and Kohn results [2] (black circles) with EDP calculated by the use of approximation 1 (e.g. (3)) with parameters given by Eqs. $(9) \div(14)$ for $r_{s}=2$ (solid line).

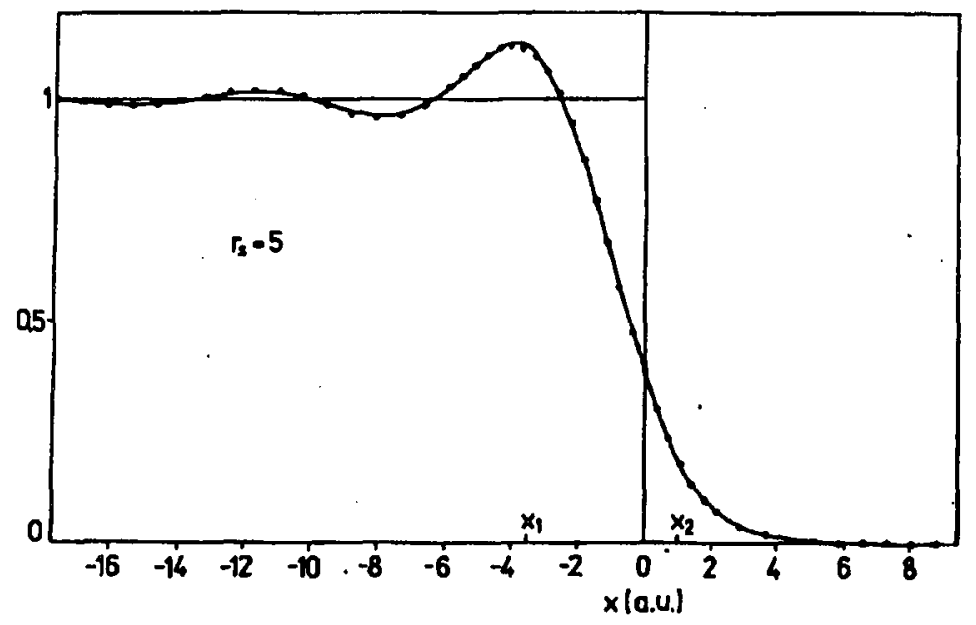

Fig. 2. The comparison of EDP obtained from Lang and Kohn results [2] (black circles) with EDP calculated by the use of approximation 2 (e.g. (3)) with parameters given by Eqs. (22) $\div(6)$ for $r_{5}=5$ (solid line).

approximation 2. Both approximations are less precise for very low and very high electron densities, i.e. for $r_{\mathrm{s}}=2$ and $r_{\mathrm{s}}=6$ than for intermediate densities. It can be also seen from Figs. 1 and 2 where EDP for $r_{\mathrm{s}}=2$ and $r_{\mathrm{s}}=5$ are given. The agreement with LK results is better for $r_{\mathrm{s}}=5$ than for $r=2$, especially in the region where the electron density oscillates.

Another way for estimation the precision of the approximation 1 and 2 is 
TABLE III

The electrostatic dipole barrier $\Delta \varphi$ and the work function (in $\mathrm{eV}$ ) for simple metals described by $r_{\mathbf{s}}$. The subscripts 1 and 2 relate to the values calculated by the use of the approximation 1 (Eqs. (9) $\div(14)$ ) and 2 (Eqs. (22) $\div(26)$ ). The subscript LK describes the results obtained self-consistently by Lang and Kohn [2], while the subscript D and K labels the values of $\varphi$ calculated analytically from DP $\Delta$ SCF $[13,14]$ and from Koopman's [12] expression (Eq. (27)), respectively.

\begin{tabular}{l|c|c|c|c|c|c|c|c}
\hline \hline$r_{\mathrm{s}}$ & $\Delta \phi_{1}$ & $\Delta \phi_{\mathrm{LK}}$ & $\Delta \phi_{2}$ & $\varphi_{\mathrm{D} 1}$ & $\varphi_{\mathrm{K} 1}$ & $\varphi_{\mathrm{LK}}$ & $\varphi_{\mathrm{D} 2}$ & $\varphi_{\mathrm{K} 2}$ \\
\hline 2 & 6.34 & 6.80 & 6.56 & 4.09 & 3.43 & 3.89 & 3.88 & 3.65 \\
2.5 & 3.69 & 3.83 & 3.72 & 3.82 & 3.57 & 3.72 & 3.72 & 3.60 \\
3 & 2.33 & 3.82 & 2.29 & 3.59 & 3.51 & 3.50 & 3.51 & 3.47 \\
3.5 & 1.53 & 1.43 & 1.48 & 3.36 & 3.35 & 3.26 & 3.31 & 3.30 \\
4 & 1.00 & 0.91 & 0.97 & 3.15 & 3.16 & 3.06 & 3.11 & 3.12 \\
4.5 & 0.64 & 0.56 & 0.63 & 2.95 & 2.95 & 2.87 & 2.93 & 2.94 \\
5 & 0.36 & 0.35 & 0.39 & 2.76 & 2.74 & 2.73 & 2.76 & 2.77 \\
5.5 & 0.15 & 0.16 & 0.21 & 2.59 & 2.54 & 2.54 & 2.60 & 2.60 \\
6 & -0.007 & 0.04 & 0.08 & 2.43 & 2.36 & 2.41 & 2.45 & 2.45
\end{tabular}

the comparison of the work functions calculated by the use of different EDP with LK results. In Table III we present results for the surface dipole barrier $\Delta \phi=$ $\phi(\infty)-\phi(-\infty)$ and the work function $\varphi$ in the density range described by $r_{\mathrm{s}}=2 \div 6$ employing the Wigner expression for the correlation energy. The work function is calculated according to the Koopmans definition [12]:

$$
\varphi_{\mathrm{K}}=\Delta \phi-\frac{1}{2} k_{F}^{2}-\frac{\mathrm{d}}{\mathrm{d} \bar{n}}\left[n \varepsilon_{\mathrm{xc}}(\bar{n})\right]
$$

and the displaced-profile change-in-selfconsistent field (DP $\triangle \mathrm{SCF}$ ) expression $[13,14]$ :

$$
\varphi_{\mathrm{D}}=\phi(\infty)-\phi(0)-\varepsilon_{\mathrm{T}} .
$$

Better agreement with LK values of work function for $r_{\mathrm{s}}<5$ gives the approximation 2 while for $r_{\mathrm{s}} \geq 5$ the approximation 1 . The above result seems to be contrary to standard deviation calculations which support approximation 1 but approximation 2 fulfills ChNC and BVT, therefore is more efficient in work function calculations.

The magnitude of electron density in the surface region plays an essential role in other physical phenomena as for example in adsorption $[15,16]$, in photoelectron spectroscopy [17] or in adhesion [18]. Adsorption or adhesion energies in contradistinction to work function depend on the tail of EDP because the binding energies are mainly determined by the overlapping of the metal and adatom or metal-metal electron clouds. In these calculations [15-18] usually the different 
TABLE IV

The values $\kappa(x)$ (the ratio of EDP given by Perdew's trial function [19] to the EDP within the approximation 1) for the values of $x$ equal to $0.1,5.0$ and $x_{\mathrm{m}}$, where $x_{\mathrm{m}}$ is the value of $x$ for maximal value of $\kappa(x)$.

\begin{tabular}{c|c|c|c|c}
\hline \hline$r_{\mathrm{s}}$ & $\kappa(0.1)$ & $\kappa_{\max }$ & $\kappa(5)$ & $x_{\mathrm{m}}$ \\
\hline 2 & 0.9743 & 1.0133 & 0.9901 & 0.90 \\
3 & 0.9915 & 1.0481 & 0.9949 & 1.30 \\
4 & 0.9908 & 1.0451 & 0.9882 & 1.90 \\
5 & 0.9844 & 1.0491 & 0.9938 & 2.50
\end{tabular}

variational trial functions are used instead of LK EDP. In order to estimate the error committed when the EDP is represented by the variational trial function $\nu_{\mathrm{P}}(x)$ we have calculated the ratio $\kappa(x)=\nu_{\mathrm{P}}(x) / \nu_{\mathrm{LK}}(x)$ in the interval $(-5,5)$, where $\nu_{\mathrm{P}}(x)$ is given by Perdew's trial [19] and $\nu_{\mathrm{LK}}(x)$ by Eq. (3) within approximation 1 . The results are presented in Table IV, where $\kappa(0.1), \kappa(5.0)$ and $\kappa_{\max }\left(x_{\mathrm{m}}\right)$, i.e. the maximal value of $\kappa(x)$, are given. They show that the ratio $\kappa(x)$ is increasing function of $r_{\mathrm{s}}$, which means that the use of the variational trial EDP gives the worst values the smaller is the mean electron density $\vec{n}$ of metal. Concluding, approximation 1 which better than approximation 2 describes the shape of EDP should be used in adsorption and adhesion energy calculations while approximation 2 is more advised in work function calculations.

\section{References}

[1] V. Heine, in: Solid State Physics, Eds. H. Ehrenreich, F. Seitz, D. Turnbull, Vol. 24, Academic Press, New York 1970, p. 1.

[2] N.D. Lang, W. Kohn, Phys. Rev. B 1, 4555 (1970).

[3] A. Kiejna, Phys. Status Solidi B 105, 147 (1981).

[4] J.M. Rogowska, K.F. Wojciechowski, H. Bogdanow, Acta Phys. Pol. A 78, 651 (1990).

[5] Theory of the Inhomogeneous Electron Gas, Eds. S. Lundquist, N.H. March, Plenum Press, New York 1983.

[6] P.J. Jennings, R.O. Jones, M. Weienert, Phys. Rev. B 37, 6113 (1988).

[7] E.E. Mola, C.A. Paola, J.L. Vicente, Phys. Rev. B 44, 13671 (1991).

[8] W. Schmickler, D. Henderson, Prog. Surf. Sci. 22, B323 (1986).

[9] H.F. Budd, J. Vannimenus, Phys. Rev. Lett. 31, 1218 (1973).

[10] A.K. Gupta, K.S. Singwi, Phys. Rev. B 15, 1801 (1977).

[11] V. Sahni, C.Q. Ma, J.S. Flamholz, Phys. Rev. B 18, 3931 (1978).

[12] N.D. Lang, W. Kohn, Phys. Rev. B 3, 1215 (1971). 
[13] R. Monnier, J.P. Perdew, D.C. Langreht, J.W. Wilkins, Phys. Rev. B 18, 656 (1978).

[14] J.P. Perdew, V. Sahni, Solid State Commun. 30, 87 (1979).

[15] J.M. Rogowska, in: Modern Problems of Surface Physics, 1st Int. School of Condensed Matter Physics, Varna 1980, Ed. I.J. Lalov, Publ. House of the Bulgarian Ac. of Sci., Sofia 1981, p. 908.

[16] J.M. Rogowska, K.F. Wojciechowski, Surf. Sci. 91.3, 422 (1989).

[17] G.K. Wertheim, D.M. Riffe, N.V.Smith, P.H. Citrin, Phys. Rev. B 46, 1955 (1992) (see also references therein).

[18] A. Kiejna, Phys. Scr. 35, 738 (1987).

[19] J.P. Perdew, Phys. Rev. B 21, 865 (1980). 